\title{
Statistical Forecasting Models of Atmospheric Carbon Dioxide and Temperature in the Middle East
}

\author{
Maryam I. Habadi*, Chris P. Tsokos \\ Department of Mathematics and Statistics, University of South Florida, Tampa, USA \\ Email: *mhabadi@mail.usf.edu
}

How to cite this paper: Habadi, M.I. and Tsokos, C.P. (2017) Statistical Forecasting Models of Atmospheric Carbon Dioxide and Temperature in the Middle East. Journal of Geoscience and Environment Protection, 5, 11-21.

https://doi.org/10.4236/gep.2017.510002

Received: August 16, 2017

Accepted: October 15, 2017

Published: October 18, 2017

Copyright $\odot 2017$ by authors and Scientific Research Publishing Inc. This work is licensed under the Creative Commons Attribution International License (CC BY 4.0).

http://creativecommons.org/licenses/by/4.0/

\begin{abstract}
Time series models are very powerful methods that help to drive hidden visions of a phenomenon and make informed future decisions. The purpose of this study is to develop statistical time series forecasting models to predict atmospheric carbon dioxide concentration in the Middle East and temperature in Saudi Arabia using multiplicative seasonal autoregressive integrated moving average models. We proceed to verify the quality and usefulness of our proposed probabilistic models by utilizing essential statistical properties to evaluate them according to their performance in forecasting the carbon dioxide in the atmosphere and the corresponding temperatures and it was shown that both statistical forecasting models produced good estimates.
\end{abstract}

\section{Keywords}

Non-Stationary Time Series, ARIMA, Global Warming

\section{Introduction}

Time series analysis is an interesting and important statistical procedure that can be used for forecasting the phenomenon of interest. This statistical method depends on tracking the phenomena (or variable) over a given time period and then predict the future based on the different values in the time series and on the pattern of growth in values. The aim of the present study is to develop statistical time series forecasting models to predict carbon dioxide $\left(\mathrm{CO}_{2}\right)$ in the atmosphere in the Middle East and atmospheric temperature in Saudi Arabia. Since it is well known that the most fundamental cause of global warming is the excessive rise of greenhouse gasses, probably the product of the industrial revolution, 
that accumulate in the atmosphere, blocking heat and leading to increased temperatures within the Earth's atmosphere. Especially, the raise proportion of the carbon dioxide from their very normal level has the most significant effect on substantial changes in the Earth's climate. The Middle East is emitting approximately 1714.09 million metric tons of carbon dioxide into the atmosphere, and based on U.S department of energy, three Middle Eastern countries are among the five highest national per capita $\mathrm{CO}_{2}$ emissions rates in the world for 2008: Qatar (14.58 metric tons of carbon per person), United Arab Emirates (9.43), and Bahrain (7.90) [1]. In a previous paper [2], we have developed a statistical model that identifies the risk factors of the atmospheric $\mathrm{CO}_{2}$ in the Middle East affected by carbon dioxide emission that is related to fossil fuels, gas flares, cement production, and their interaction terms. We have found that gas-fuels, liquid fuels, cement, and only 4 interaction terms namely (Liquid Fuels ${ }^{\star}$ Solid Fuels), (Liquid Fuels ${ }^{\star}$ Gas Flares), (Solid Fuels ${ }^{\star}$ Cement) and (Gas Flares ${ }^{\star}$ Cement) are significantly contributing to atmospheric $\mathrm{CO}_{2}$ in the Middle East, as well as statistical models of carbon dioxide in the atmosphere in the United States, Europe and South Korea [3]-[8]. Thus, the objective of the present study is to develop two different statistical time series forecasting models for the atmospheric carbon dioxide concentration in the Middle East, in addition to atmospheric temperature in Saudi Arabia.

\section{Atmospheric $\mathrm{CO}_{2}$ Statistical Forecasting Model}

To develop our statistical forecasting model, we used monthly data of atmospheric carbon dioxide concentrations measured in part per million from 1996 to 2015. The data was collected in Weizmann Institute of science at the Arava Institute and provided by National Oceanic and Atmospheric Administration, Earth system research laboratory, Global Monitoring Division, Boulder, Colorado, USA (https://esrl.noaa.gov/gmd/). Figure 1 below gives a visual presentation of the time series plot of atmospheric $\mathrm{CO}_{2}$ in the Middle East.

The data is clearly non-stationary with seasonality and increasing trend. Most

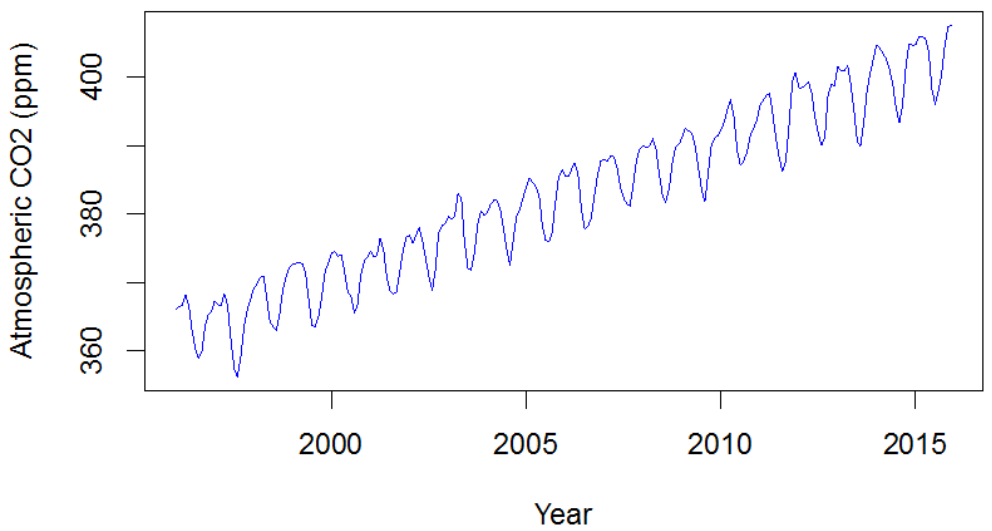

Figure 1. Time series plot of the atmospheric $\mathrm{CO}_{2}$ data in the Middle East from 1996-2015. 
of the time series we encounter in real world problems are non-stationary, and we must remove non-stationary component to utilize methodology for stationary time series data. Thus, in order for us to do the analysis, we must first reduce a non-stationary time series into a stationary time series after applying a proper degree of difference filter of the given series. Since we have a seasonal data, the multiplicative seasonal autoregressive integrated moving average (seasonal ARIMA) model will be used to develop the statistical predictive model of the atmospheric carbon dioxide in the Middle East [9] [10] [11]. A seasonal ARIMA model is formed by including seasonal terms in the autoregressive integrated moving average model $\operatorname{ARIMA}(p, d, q)$ as is defined as follows

$$
\phi_{p}(B)(1-B)^{d} x_{t}=\theta_{q}(B) \varepsilon_{t}
$$

where $p$ is order of autoregressive process, $d$ is degree of differencing (filter); $q$ is order of moving average, and the analytical form of seasonal $\operatorname{ARIMA}(p, d, q)(P, D, Q)_{s}$ is defined by

$$
\Phi_{P}\left(B^{S}\right) \phi_{p}(B)(1-B)^{d}\left(1-B^{S}\right)^{D} x_{t}=\theta_{q}(B) \Theta_{Q}\left(B^{S}\right) \varepsilon_{t}
$$

where $p, d$ and $q$ as defined above, also , $P$ is the order of the seasonal autoregressive process, $D$ is the order of the seasonal differencing, $Q$ is the order of the seasonal moving average, and the subindex $S$ refers to the seasonal period, with monthly data $S=12$; for quarterly data $S=4$, and $\Phi_{P}\left(B^{S}\right), \phi_{p}(B), \theta_{q}(B), \Theta_{Q}\left(B^{S}\right)$ are defined as follows:

The non-seasonal components we have:

$$
\text { AR : } \phi_{p}(B)=\left(1-\phi_{1} B-\phi_{2} B^{2}-\cdots-\phi_{p} B^{p}\right)
$$

and

$$
\mathrm{MA}: \theta_{q}(B)=\left(1+\theta_{1} B+\theta_{2} B^{2}+\cdots+\theta_{q} B\right)
$$

The seasonal components are:

$$
\text { Seasonal AR : } \Phi_{P}\left(B^{S}\right)=\left(1-\Phi_{1} B^{S}-\Phi_{2} B^{2 S}-\cdots-\Phi_{P} B^{P S}\right)
$$

and

$$
\text { Seasonal MA : } \Theta_{Q}\left(B^{S}\right)=\left(1+\Theta_{1} B^{S}+\Theta_{2} B^{2 S}+\cdots+\Theta_{Q} B^{Q S}\right)
$$

In the present study, since we have a monthly data, we let the seasonal subin$\operatorname{dex} S=12$. Once we transform our data into stationary time series, we found that the best statistical forecasting model that characterizes the monthly atmospheric carbon dioxide concentration in the Middle East with minimum AIC [12] is $\operatorname{ARIMA}(2,1,3)(0,1,1)_{12}$; analytically is given by

$$
\begin{aligned}
& \left(1-\phi_{1} B-\phi_{2} B^{2}\right)(1-B)\left(1-B^{12}\right) x_{t} \\
& =\left(1+\theta_{1} B+\theta_{2} B^{2}+\theta_{3} B^{3}\right)\left(1+\Theta_{1} B^{12}\right) \varepsilon_{t}
\end{aligned}
$$

with first non-seasonal difference filter and first seasonal difference filter, second order of non-seasonal autoregressive process $\mathrm{AR}(2)$, third order of non-seasonal moving average process $\mathrm{MA}(3)$, and first order of seasonal moving average 
process SMA(1). Expanding both sides of the above ARIMA model, we have

$$
\begin{aligned}
& {\left[1-\left(1+\phi_{1}\right) B+\left(\phi_{1}-\phi_{2}\right) B^{2}+\phi_{2} B^{3}-B^{12}\right.} \\
& \left.+\left(1+\phi_{1}\right) B^{13}+\left(\phi_{2}-\phi_{1}\right) B^{14}-\phi_{2} B^{15}\right] x_{t} \\
& =\left[1+\theta_{1} B+\theta_{2} B^{2}+\theta_{3} B^{3}+\Theta_{1} B^{12}+\theta_{1} \Theta_{1} B^{13}+\theta_{2} \Theta_{1} B^{14}+\theta_{3} \Theta_{1} B^{15}\right] \varepsilon_{t}
\end{aligned}
$$

Simplify it and using backshift operation $B^{j} x_{t}=x_{t-j}$, we obtain

$$
\begin{aligned}
x_{t}= & \left(1+\phi_{1}\right) x_{t-1}-\left(\phi_{1}-\phi_{2}\right) x_{t-2}-\phi_{2} x_{t-3}+x_{t-12}-\left(1+\phi_{1}\right) x_{t-13} \\
& -\left(\phi_{2}-\phi_{1}\right) x_{t-14}+\phi_{2} x_{t-15}+\varepsilon_{t}+\theta_{1} \varepsilon_{t-1}+\theta_{2} \varepsilon_{t-2}+\theta_{3} \varepsilon_{t-3} \\
& +\Theta_{1} \varepsilon_{t-12}+\theta_{1} \Theta_{1} \varepsilon_{t-13}+\theta_{2} \Theta_{1} \varepsilon_{t-14}+\theta_{3} \Theta_{1} \varepsilon_{t-15}
\end{aligned}
$$

Thus, the approximate maximum likelihood estimates of the coefficients are

$$
\begin{gathered}
\phi_{1}=-0.6791, \phi_{2}=0.1376, \theta_{1}=0.9140 \\
\theta_{2}=-0.8964, \theta_{3}=-0.8803, \Theta_{1}=-0.9996
\end{gathered}
$$

by letting $\varepsilon_{t}=0$, the one-step ahead forecasting model for atmospheric $\mathrm{CO}_{2}$ in the Middle East is given by

$$
\begin{aligned}
\hat{x}_{t}= & 0.3209 x_{t-1}+0.8167 x_{t-2}-0.1376 x_{t-3}+x_{t-12}-0.3209 x_{t-13}-0.8167 x_{t-14} \\
& +0.1376 x_{t-15}+0.9140 \varepsilon_{t-1}-0.8964 \varepsilon_{t-2}-0.8803 \varepsilon_{t-3} \\
& -0.9996 \varepsilon_{t-12}-0.9136 \varepsilon_{t-13}+0.8960 \varepsilon_{t-14}+0.8799 \varepsilon_{t-15}
\end{aligned}
$$

Once we identify the forecasting model of the atmospheric carbon dioxide, we need to evaluate or validate our proposed model and illustrate the quality of model. In Figure 2 below presents the actual data with the forecasting values of the atmospheric carbon dioxide in the Middle East that obtained by our proposed statistical forecasting model. In addition, we perform residual analysis and calculate the residuals estimates $r_{t}=x_{t}-\hat{x}_{t}$; Figure 3 below shows the graphical result of the residual estimates.

We can see in Figure 2, the predicted values follow the original data of the atmospheric $\mathrm{CO}_{2}$. Furthermore, the residuals in Figure 3 are quite small and isolating around zero and that is an indication of the good quality of our proposed statistical time series-forecasting model of the atmospheric $\mathrm{CO}_{2}$ in the Middle East. Next, we evaluate the mean of the residuals, $\bar{r}$, the variance, $S_{r}^{2}$, and the mean square error, MSE, and the results are presented in Table 1.

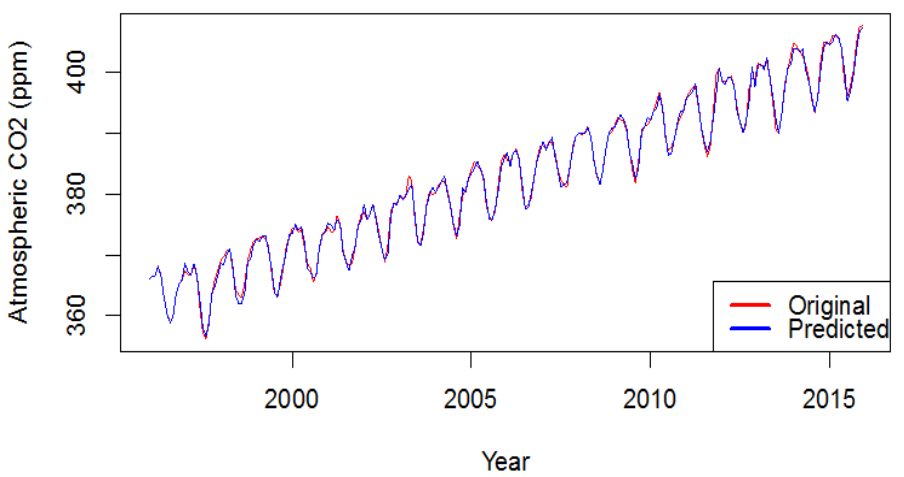

Figure 2. Original vs. predicted values of atmospheric $\mathrm{CO}_{2}$. 
Table 1. Basic Evaluation on atmospheric carbon dioxide model.

\begin{tabular}{ccc}
\hline $\bar{r}$ & $S_{r}^{2}$ & MSE \\
\hline 0.0812 & 0.5062 & 0.5107 \\
\hline
\end{tabular}

The results show the effectiveness of the proposed model for forecasting atmospheric carbon dioxide in the Middle East.

Furthermore, we restructure the model (6) with monthly data from 1996-2013 to forecast the last 24 hidden values of using the previous observations. The purpose is to test the accuracy of the forecasting values of the atmospheric $\mathrm{CO}_{2}$ with respect to the observed 24 values that have not been used and how well the model performs on new data that were not used when fitting the model. Table 2 gives the actual and predicted values of carbon dioxide in the atmosphere.

As we can see, the difference between the original and predicted values of the carbon dioxide in the Middle East is very small. Figure 4 gives a graphical presentation of the results in Table 2.

Since the predicted values produced by our proposed statistical model are very close to the original values, and the forecast errors seem to be very small, the $\operatorname{ARMA}(2,1,3)(0,1,1)_{12}$ does seem to provide an adequate predictive model for the atmospheric carbon dioxide in the Middle East.

Table 2. Actual vs. Forecasting values of Atmospheric $\mathrm{CO}_{2}$.

\begin{tabular}{cccc}
\hline Year & Original values & Forecasting values & Residuals \\
\hline Jan 2014 & 404.75 & 403.76 & 0.99 \\
Feb 2014 & 404.12 & 402.60 & 1.52 \\
Mar 2104 & 403.38 & 402.55 & 0.83 \\
Apr 2104 & 402.58 & 403.45 & -0.87 \\
May 2104 & 400.97 & 401.43 & -0.46 \\
Jun 2104 & 398.95 & 397.63 & 1.32 \\
Jul 2104 & 395.35 & 394.90 & 0.45 \\
Aug 2104 & 393.36 & 393.97 & -0.61 \\
Sep 2104 & 395.86 & 395.76 & 0.10 \\
Oct 2014 & 401.45 & 399.85 & 1.60 \\
Nov 2014 & 404.86 & 402.25 & 2.61 \\
Dec 2104 & 404.63 & 403.31 & 1.32 \\
Jan 2015 & 404.69 & 404.16 & 0.53 \\
Feb 2105 & 405.92 & 404.30 & 1.62 \\
Mar 2015 & 405.92 & 404.60 & 1.32 \\
Apr 2015 & 405.6 & 405.44 & 0.16 \\
May 2015 & 403.84 & 403.51 & 0.33 \\
Jun 2015 & 398.26 & 399.64 & -1.38 \\
Jul 2015 & 396.02 & 396.97 & -0.95 \\
Aug 2015 & 397.86 & 395.98 & 1.88 \\
Sep 2105 & 400.33 & 397.83 & 2.50 \\
Oct 2015 & 404.64 & 401.88 & 2.76 \\
Nov 2015 & 407.33 & 404.31 & 3.02 \\
Dec 2105 & 407.54 & 405.33 & 2.21 \\
\hline & & & \\
\hline
\end{tabular}




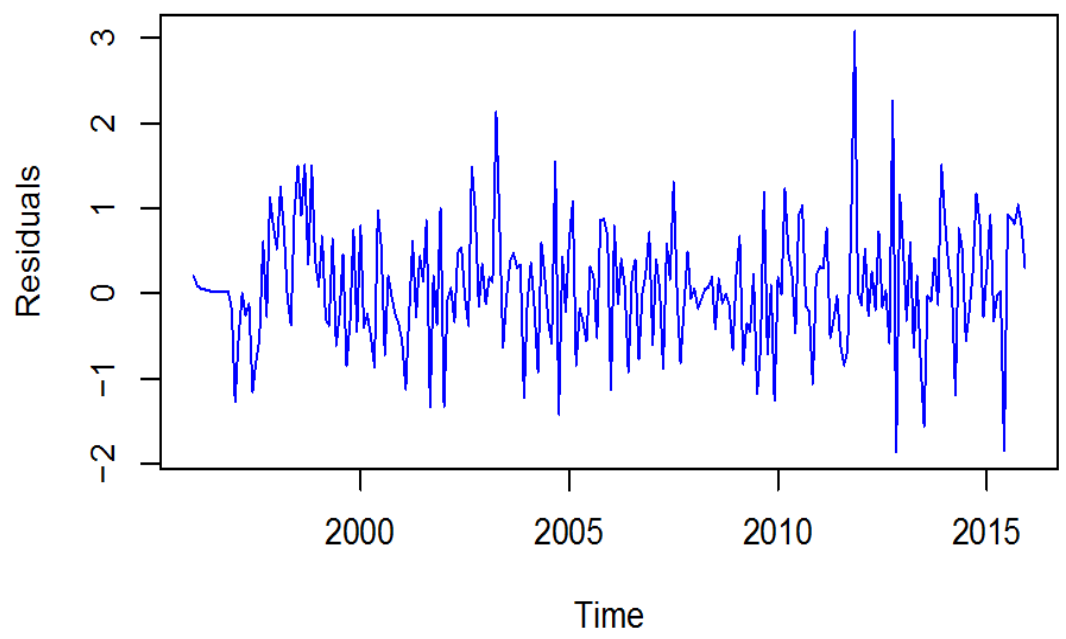

Figure 3. Residual plot of monthly atmospheric carbon dioxide.

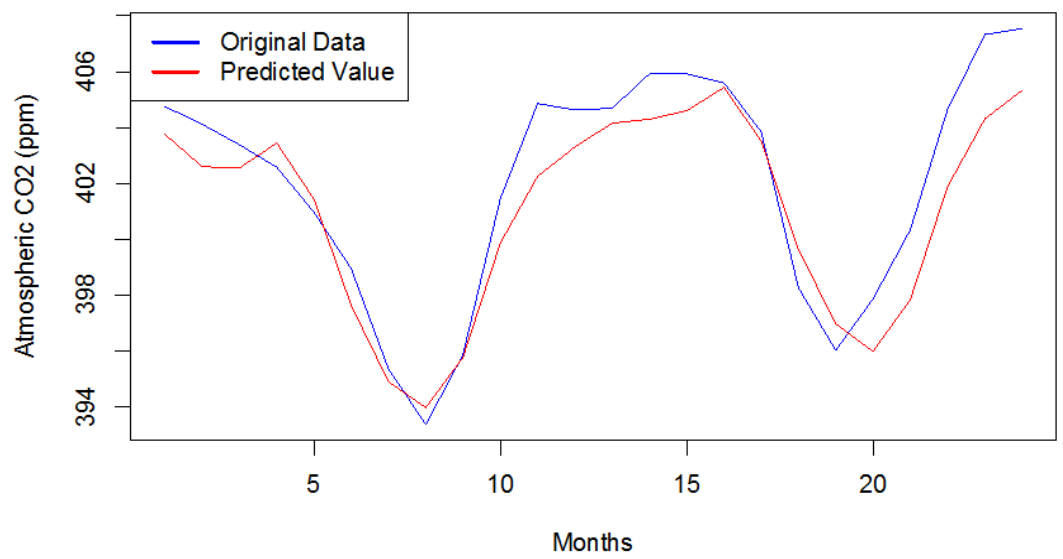

Figure 4. Monthly atmospheric $\mathrm{CO} 2$ vs. predicted values for the last 24 months.

\section{Atmospheric Temperature Forecasting Model of Saudi Arabia}

Saudi Arabia's prevailing climate is hot and dry, but according to weather expert, The Kingdom of Saudi Arabia has witnessed an unprecedented drop in temperature accompanied by uncommon natural phenomena. Frost and freezing temperatures and unusually heavy snowfall have been reported in several areas in Saudi Arabia in winter, as well as increasing the heat in summer. In general, the changes in the global climate due to the impact of global warming will lead tomore extreme seasons. Thus, the aim of this part is to develop a statistical forecasting model for temperature in Saudi Arabia as temperature plays an important role in Global warming.

The dataset includes monthly average temperature measured in Celsius $\left({ }^{\circ} \mathrm{C}\right)$ of Saudi Arabia as only available data from January 1970 to December 2015. The data was published by the Saudi's General Authority of Meteorology and Environmental protection. A presentation of the temperature data is given in Figure 5. 


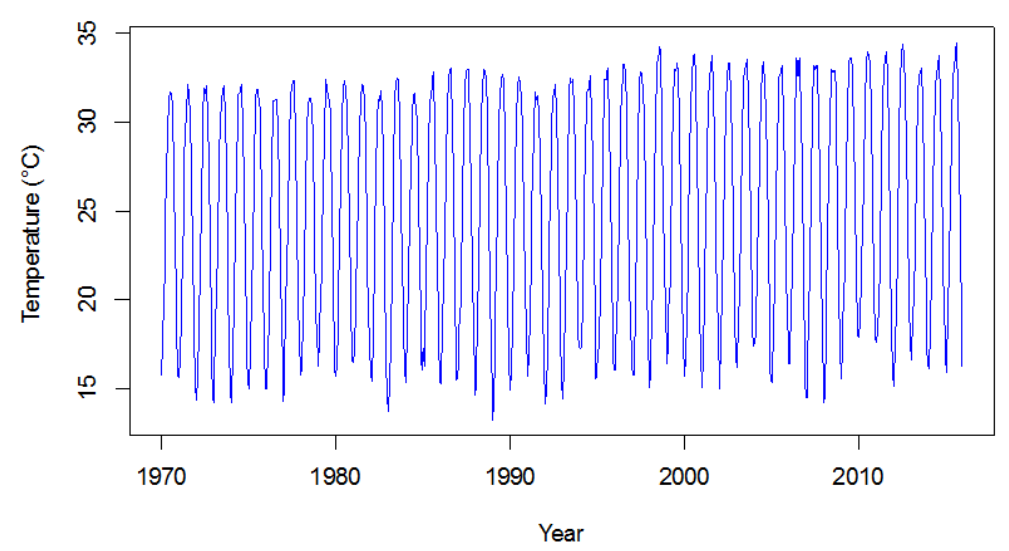

Figure 5. Time series plot of monthly temperature from 1970-2015.

We will develop a forecasting model using the multiplicative seasonal autoregressive integrated moving average (seasonal ARIMA) model as described in section 2 [13] [14] [15]. Thus, after confirming the stationary of our series and let the seasonal subindex $S=12$, we found the model that best described the monthly atmospheric temperature of the kingdom of Saudi Arabia is $\operatorname{ARIMA}(1,1,2)(0,1,1)_{12}$, and analytically is given by

$$
\left(1-\phi_{1} B\right)(1-B)\left(1-B^{12}\right) x_{t}=\left(1+\theta_{1} B+\theta_{2} B^{2}\right)\left(1+\Theta_{1} B^{12}\right) \varepsilon_{t},
$$

with first non-seasonal difference filter and first seasonal difference filter, first order of non-seasonal autoregressive process $\mathrm{AR}(1)$, second order of non-seasonal moving average process $\mathrm{MA}(2)$, and first order of seasonal moving average process SMA(1). Expanding both sides, we have

$$
\begin{aligned}
& {\left[1-\left(1+\phi_{1}\right) B+\phi_{1} B^{2}-B^{12}+\left(1+\phi_{1}\right) B^{13}-\phi_{1} B^{14}\right] x_{t}} \\
& =\left[1+\theta_{1} B+\theta_{2} B^{2}+\Theta_{1} B^{12}+\theta_{1} \Theta_{1} B^{13}+\theta_{2} \Theta_{1} B^{14}\right] \varepsilon_{t}
\end{aligned}
$$

Simplify it, we get

$$
\begin{aligned}
& x_{t}-\left(1+\phi_{1}\right) x_{t-1}+\phi_{1} x_{t-2}-x_{t-12}+\left(1+\phi_{1}\right) x_{t-13}-\phi_{1} x_{t-14} \\
& =\varepsilon_{t}+\theta_{1} \varepsilon_{t-1}+\theta_{2} \varepsilon_{t-2}+\Theta_{1} \varepsilon_{t-12}+\theta_{1} \Theta_{1} \varepsilon_{t-13}+\theta_{2} \Theta_{1} \varepsilon_{t-14}
\end{aligned}
$$

The approximate maximum likelihood estimates of the coefficients are

$$
\phi_{1}=0.6546, \theta_{1}=-1.3691, \theta_{2}=0.3706, \Theta_{1}=-0.9785
$$

Thus, the forecasting model for the monthly atmospheric temperature of Saudi Arabia is given by

$$
\begin{aligned}
\hat{x}_{t}= & 1.6546 x_{t-1}-0.6546 x_{t-2}+x_{t-12}-1.6546 x_{t-13}+0.6546 x_{t-14} \\
& -1.3691 \varepsilon_{t-1}+0.3706 \varepsilon_{t-2}-0.9785 \varepsilon_{t-12}+1.3396 \varepsilon_{t-13}-0.3626 \varepsilon_{t-14}
\end{aligned}
$$

To examine the quality of our proposed model, first we graph the forecasting values obtained by our proposed $\operatorname{ARIMA}(1,1,2)(0,1,1)_{12}$ model on the top of the original time series data as shown in Figure 6.

As we can see, the predicted values follow the actual data of the monthly temperature of Saudi Arabia and that an indication of good quality of our proposed 
forecasting model.

Next, we calculate the residuals estimate and evaluate the mean of the residuals, $\bar{r}$, the variance, $S_{r}^{2}$, and the mean square error, MSE. The results are presented in Table 3; Figure 7 shows a graphical presentation of the residual estimates.

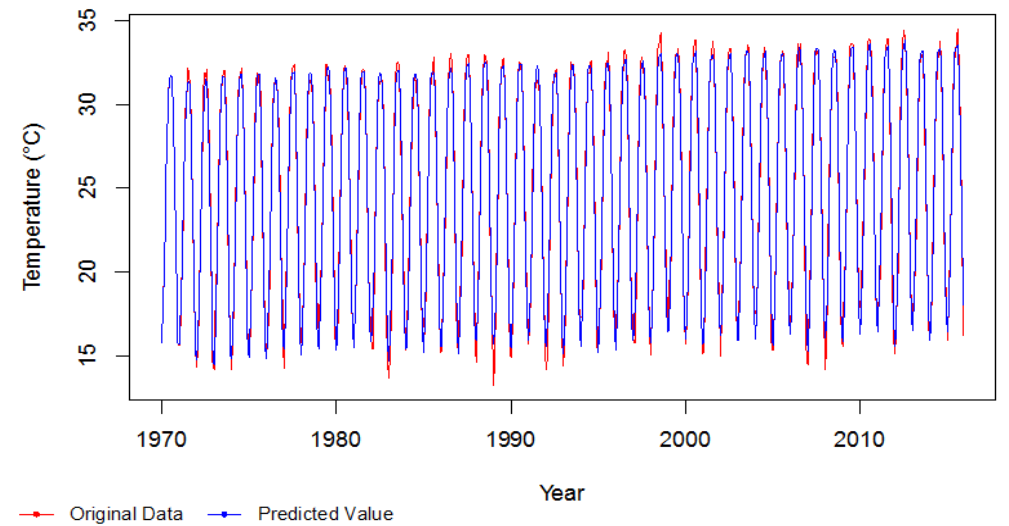

Figure 6. Original vs. predicted values of monthly temperature.

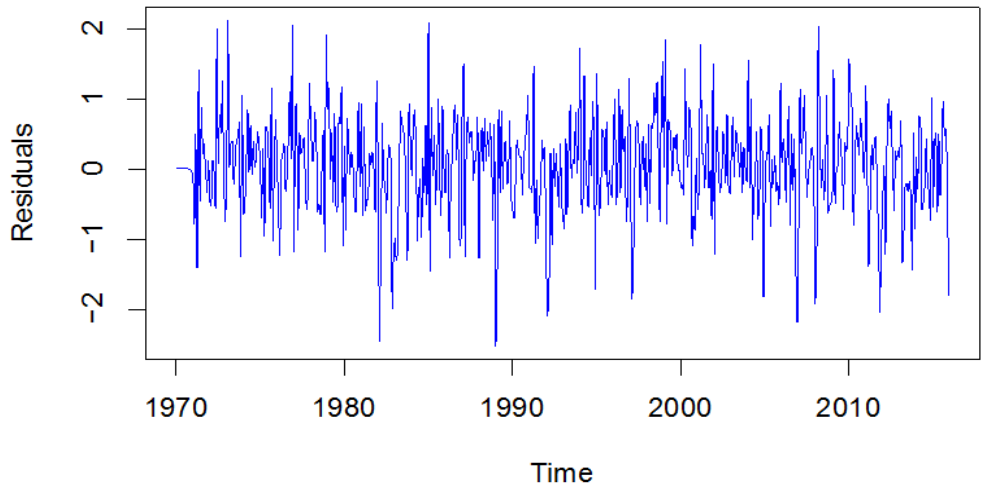

Figure 7. Residual plot for monthly temperature of Saudi Arabia.

Table 3. Basic evaluation on temperature model.

\begin{tabular}{ccc}
\hline $\bar{r}$ & $S_{r}^{2}$ & MSE \\
0.0451 & 0.5366 & 0.5376 \\
\hline
\end{tabular}

The mean of the residuals is very close to zero and it illustrates the best quality of the model, in addition, the residual plot in Figure 7 shows that the residual estimated of our proposed model are very small and isolating around zero and the variation of the residuals stays much the same across the time series data. These results also support the effectiveness of the proposed model for forecasting average monthly atmospheric temperature in Saudi Arabia.

Moreover, we restructure model (10) again using portion of the data for fitting, and use the rest of the data for testing the model. The testing data can be used to measure how well the model is likely to forecast on new data. Table 4 
gives the 24 hidden values of average monthly temperature, predicted values, and the residuals.

The average of these residuals is $\bar{r}=0.0931$, and Figure 8 shows a graphical result of the predicted values of the average monthly temperature using our proposed forecasting model.

Notice how well the forecasts follow the trend in the original data of the average atmospheric temperature in Saudi Arabia, and that is another evidence of the good quality of our proposed forecasting model.

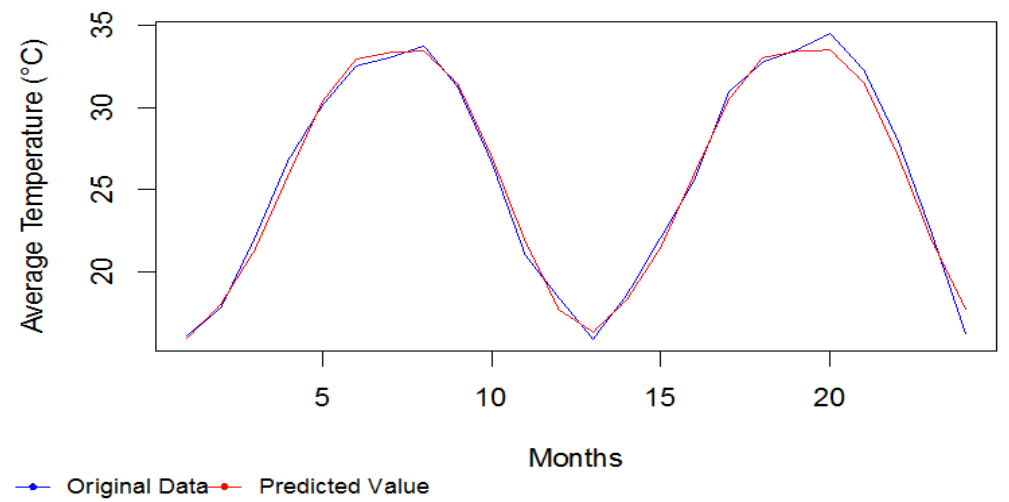

Figure 8. Original data vs. forecasts of the average temperature.

Table 4. Original data vs. forecasting values of average temperature.

\begin{tabular}{cccc}
\hline Year & Original values & Forecasting values & Residuals \\
\hline Jan 2014 & 16.092 & 15.91702 & 0.17498 \\
Feb 2014 & 17.7728 & 18.01089 & -0.23809 \\
Mar 2104 & 22.0042 & 21.28802 & 0.71618 \\
Apr 2104 & 26.802 & 25.87299 & 0.92901 \\
May 2104 & 30.1104 & 30.35522 & -0.24482 \\
Jun 2104 & 32.5334 & 32.94024 & -0.40684 \\
Jul 2104 & 33.0339 & 33.34407 & -0.31017 \\
Aug 2104 & 33.7543 & 33.44256 & 0.31174 \\
Sep 2104 & 31.2507 & 31.43814 & -0.18744 \\
Oct 2014 & 26.689 & 27.03107 & -0.34207 \\
Nov 2014 & 21.0057 & 21.84871 & -0.84301 \\
Dec 2104 & 18.3979 & 17.66596 & 0.73194 \\
Jan 2015 & 15.9007 & 16.33336 & -0.43266 \\
Feb 2105 & 18.6084 & 18.30048 & 0.30792 \\
Mar 2015 & 22.0948 & 21.49318 & 0.60162 \\
Apr 2015 & 25.6254 & 26.0219 & -0.3965 \\
May 2015 & 30.9111 & 30.46668 & 0.44442 \\
Jun 2015 & 32.7563 & 33.02674 & -0.27044 \\
Jul 2015 & 33.5017 & 33.41394 & 0.08776 \\
Aug 2015 & 34.5059 & 33.50136 & 1.00454 \\
Sep 2105 & 32.2554 & 31.48957 & 0.76583 \\
Oct 2015 & 27.9791 & 27.07759 & 0.90151 \\
Nov 2015 & 22.3221 & 21.89195 & 0.43015 \\
Dec 2105 & 16.2065 & 17.70703 & -1.50053 \\
\hline
\end{tabular}




\section{Conclusion}

In the present study, we have developed two seasonal autoregressive integrated moving average models to forecast the monthly atmospheric carbon dioxideconcentration in the Middle East and monthly average atmospheric temperature in Saudi Arabia. The two developed statistical forecasting models were evaluated using different statistical criteria; also tested the accuracy of the predicted values and it was shown that both statistical forecasting models produced good estimates.

\section{References}

[1] Boden, T.A., Marland, G. and Andres, R.J. (2011) Global, Regional, and National Fossil-Fuel $\mathrm{CO}_{2}$ Emissions. Carbon Dioxide Information Analysis Center, Oak Ridge National Laboratory, U.S. Department of Energy, Oak Ridge.

[2] Habadi, M.I. and Tsokos, C.P. (2016) Statistical Analysis and Modeling of the Atmospheric Carbon Dioxide in the Middle East and Comparisons with USA, EU and South Korea. SCIREA Journal of Environment, 1, 32-47.

[3] Kim, D. and Tsokos, C.P. (2013) Statistical Significance of Fossil Fuels Contributing to Atmospheric Carbon Dioxide in South Korea and Comparisons with USA and EU. Journal of Applied Statistical Science, 21, 337.

[4] Wooten, R.D. and Tsokos, C.P. (2010) Parametric Analysis of Carbon Dioxide in the Atmosphere. Journal of Applied Sciences, 10, 440-450.

https://doi.org/10.3923/jas.2010.440.450

[5] Shih, S.H. and Tsokos, C.P. (2008) Prediction Models for Carbon Dioxide Emissions and the Atmosphere, to Appear. The International Journal Neural, Parallel \& Scientific Computations, 16, 165-178.

[6] Xu, Y. and Tsokos, C.P. (2013) Attributable Variables with Interactions That Contribute to Carbon Dioxide in the Atmoshpere. Frontiers in Science, 3, 6-13.

[7] Xu, Y. and Tsokos, C.P. (2011) Statistical Models and Analysis of Carbon Dioxide in the Atmosphere. Problems of Nonlinear Analysis in Engineering Systems, 2, e1.

[8] Teodorescu, I. and Tsokos, C. (2013) Contributors of Carbon Dioxide in the Atmosphere in Europe. arXiv Preprint arXiv:1312.7867.

[9] Tsokos, C.P. (1973) Forecasting Models from Non-Stationary Time Series-Short Term Predictability of Stocks. Mathematical Methods in Investment and Finance. North Holland Publishing Co., Amsterdam, 520-563.

[10] Box, G.E., Jenkins, G.M., Reinsel, G.C. and Ljung, G.M. (2015) Time Series Analysis: Forecasting and Control. John Wiley \& Sons, Hoboken.

[11] Hyndman, R.J. and Athanasopoulos, G. (2013) Forecasting: Principles and Practice. https://www.otexts.org/fpp/

[12] Akaike, H. (1974) A New Look at the Statistical Model Identification. IEEE Transactions on Automatic Control, AC-19, 716-723.

https://doi.org/10.1109/TAC.1974.1100705

[13] Shih, S. H. and Tsokos, C.P. (2008) A Temperature Forecasting Model for the Continental United States, to Appear, The International Journal Neural, Parallel \& Scientific Computations, 16, 165.

[14] Shih, S.H. and Koutras, D. (2007) Analytical Model for Economic Forecasting, to Appear, Proceedings of the 5th International Conference on Dynamic Systems and 
Applications.

[15] Shih, S.H. and Tsokos, C.P. (2007) New Nonstationary Time Series Models with Economic Applications, to Appear, Proceedings of The 5th International Conference on Dynamic Systems and Applications. 